\title{
Robot-Assisted Training of Arm and Hand Movement Shows Functional Improvements for Incomplete Cervical Spinal Cord Injury
}

\author{
Gerard E. Francisco, MD, Nuray Yozbatiran, PhD, Jeffrey Berliner, DO, Marcia K. O'Malley, PhD, \\ Ali Utku Pehlivan, PhD, Zahra Kadivar, PhD, Kyle Fitle, MS, and Corwin Boake, PhD
}

Objective: The aim of the study was to demonstrate the feasibility, tolerability, and effectiveness of robotic-assisted arm training in incomplete chronic tetraplegia.

Design: Pretest/posttest/follow-up was conducted. Ten individuals with chronic cervical spinal cord injury were enrolled. Participants performed single degree-of-freedom exercise of upper limbs at an intensity of 3-hr per session for 3 times a week for 4 wks with MAHI Exo-II. Arm and hand function tests (Jebsen-Taylor Hand Function Test, Action Research Arm Test), strength of upper limb (upper limb motor score, grip, and pinch strength), and independence in daily living activities (Spinal Cord Independence Measure II) were performed at baseline, end of training, and 6 mos later.

Results: After 12 sessions of training, improvements in arm and hand functions were observed. Jebsen-Taylor Hand Function Test (0.14 [0.04]-0.21 [0.07] items/sec, $P=0.04)$, Action Research Arm Test (30.7 [3.8]-34.3 [4], $P=0.02)$, American Spinal Injury Association upper limb motor score (31.5 [2.3]-34 [2.3], $P=0.04)$ grip (9.7 [3.8]-12 [4.3] lb, $P=0.02)$, and pinch strength (4.5 [1.1]-5.7[1.2] lb, $P=0.01)$ resulted in significant increases. Some gains were maintained at 6 mos. No change in Spinal Cord Independence Measure II scores and no adverse events were observed.

Conclusions: Results from this pilot study suggest that repetitive training of arm movements with MAHI Exo-II exoskeleton is safe and has potential to be an adjunct treatment modality in rehabilitation of persons with spinal cord injury with mild to moderate impaired arm functions.

Key Words: Spinal Cord Injury, Robotic-Assisted Training, Upper Limb, Functional Recovery

(Am J Phys Med Rehabil 2017;96(Suppl):S171-S177)

$T_{\mathrm{d}}^{\mathrm{e}}$ etraplegia caused by injury to spinal cord has a significant disabling effect on independence in daily life. Approximately half of people with tetraplegia reported regaining arm and hand functions as the most important factor to improve their quality of life. ${ }^{1,2}$ Treatment options aiming to improve upper limb motor functions are sparse; functional electrical stimulation $^{3}$ and exercise ${ }^{4}$ are aimed at sensory-motor recovery,

From the Department of Physical Medicine and Rehabilitation, University of Texas Health Science Center McGovern Medical School at Houston and the NeuroRecovery Research Center at TIRR Memorial Hermann, Houston, Texas (GEF, NY, JB, CB); Department of Mechanical Engineering, Rice University, Houston, Texas (MKO, AUP, ZK, KF); and The Institute for Rehabilitation and Research/Memorial Hermann, Houston, Texas (MKO).

All correspondence and requests for reprints should be addressed to: Gerard E. Francisco, MD, TIRR Memorial Hermann, 1333 Moursund Street, Houston, TX 77030 .

This study has been funded by The Institute for Rehabilitation and Research Foundation/Mission Connect.

A case report from the study was previously published in Journal of Rehabilitation Medicine $(2012 ; 44(2): 186-8)$ and presented in poster form at International Conference on Rehabilitation Robotics 2011 (June 29-July 1, 2011, Zurich, Switzerland), International Neurorehabilitation Symposium 2011 (June 27-29, 2011, Zurich, Switzerland), and Academy of Spinal Cord Injury Professionals 2011 (September 5-7, 2011, Las Vegas, Nevada).

Financial disclosure statements have been obtained, and no conflicts of interest have been reported by the authors or by any individuals in control of the content of this article.

Supplemental digital content is available for this article. Direct URL citations appea in the printed text and are provided in the HTML and PDF versions of this article on the journal's Web site (www.ajpmr.com).

Copyright (C) 2017 Wolters Kluwer Health, Inc. All rights reserved.

ISSN: 0894-9115

DOI: 10.1097/PHM.0000000000000815 whereas other treatments offer functional gains with minimal or no effects on neurorecovery. For example, neuroprostheses ${ }^{5}$ and brain computer interface systems ${ }^{6}$ increase motor control through alternative communication and control systems, whereas reconstructive surgery of the upper limb offers permanent changes to muscle structure. ${ }^{7}$ There is evidence that repetitive and intensive practice can induce practice-dependent brain and spinal plasticity and that exercise intensity has a profound effect on sensory-motor recovery of patients with spinal cord injury (SCI). ${ }^{8,9}$ In this regard, rehabilitation robots hold promise for enhancing traditional physical and/or occupational therapy. They can deliver repetitive exercises at high intensities, for extended time periods, in a consistent and precise manner. In addition, real-time measurement of performance may provide advantage to therapists to modify the therapy protocol based on improvement in performance. In this context, previous studies have reported that robotic-assisted rehabilitation can improve motor recovery after stroke and that robotic devices are safe and feasible in rehabilitation. ${ }^{10}$ Despite considerable interest in robotic gait training after SCI, ${ }^{1,12}$ very few reports have evaluated the effect of robotic training of arm and hand function in patients with tetraplegia. ${ }^{13-15}$

The current study aimed to demonstrate the feasibility and tolerability and to assess effectiveness of robotic-assisted training in improving arm and hand functions in chronic, incomplete cervical SCI. We hypothesized that intense active repetitive movement training, with a robotic device, would 
improve arm and hand motor function in patients with chronic tetraplegia due to traumatic SCI. In addition, we aimed to evaluate the therapeutic potential of the MAHI Exo-II robotic device (Rice University, Houston, TX) in mild or severe arm paralysis. Injury to spinal cord often produces bilateral arm weakness with resulting asymmetrical degree-of-impairment in voluntary movement control between right and left arms. For example, one side can present stronger motor function while the other arm may have moderate to severe function loss. The MAHI Exo-II allows therapy to be delivered to both arms with various degrees of impairment, and results are presented separately for less and more affected arms. The current pilot study is the first aiming to train both upper limbs with a robotic device, in chronic incomplete cervical SCI.

\section{METHODS}

Ten adults ( 8 men, 2 women; mean [SEM] age $=40$ [21] yrs; mean $[\mathrm{SEM}]$ time post-SCI $=49[62] \mathrm{mos}$ ) with chronic, cervical SCI, referred from The Institute for Rehabilitation and Research Memorial Hermann, participated in the study. Study participants were between 19 and 76 yrs old and were diagnosed with injury level between $\mathrm{C} 2$ and $\mathrm{C} 7$ and impairment level between American Spinal Injury Association (ASIA) B-D. Table 1 summarizes the characteristics of the study group. Enrolled subjects had to have diagnosis of a complete or incomplete cervical lesion as defined by the ASIA classification at the time of injury, upper limb weakness associated with tetraplegia, no previous central or peripheral nervous system insult interfering with interpretation of the results, English-language comprehension and cognitive ability sufficient to cooperate with the intervention, no joint contracture or severe spasticity in the affected upper limb, as measured by a Modified Ashworth Scale score higher than 3 of 4 , no involvement in any specific exercise program within the previous 3 mos and no planned alteration in upper limb therapy or medication for muscle tone during the course of the study, no recent significant trauma to forearm or hand, eligibility for standard rehabilitation at the time of enrollment (i.e., absence medical co-morbidities that would prevent standard rehabilitation), and no condition (e.g., severe arthritis, extreme shoulder pain) that would interfere with valid administration of the measures. Subjects were excluded if they had inability to undergo screening for eligibility, baseline testing, and were not able to provide self-transportation to study location.
Study related procedures were explained in detail by a researcher, and each subject provided written informed consent in accordance with University of Texas Health Science Center at Houston, Institutional Review Board.

\section{Assessment}

Subjects underwent outcome assessment before treatment, immediately after treatment and at 6-mo follow-up. To avoid intertester variability, the same therapist who had more than $10 \mathrm{yrs}$ of experience working with neurologically impaired patients performed all tests. At the time of enrollment, all subjects had minimal movement in one or both upper limbs.

\section{Testing of Arm and Hand Muscle Strength and Functions for Efficacy}

To evaluate the effect of robotic training on arm and hand motor functions, test scores from right and left limb were averaged (except for ASIA-Upper Extremity Motor Score [UEMS]) and used for analysis. Secondarily, each limb was assigned into two different groups that differed according to the level of impairment. The limb with higher baseline ASIA-UEMS was defined as less affected, whereas the other limb was defined as more affected.

\section{Testing for Arm Strength}

Strength of selected muscles (C5-T1: biceps, triceps, wrist extensor, finger flexor, finger abductor) in both upper limb was scored using the Medical Research Council grade $(0=$ absent, $5=$ normal $)$ in accordance with ASIA guidelines. ${ }^{16}$ Despite standardized body supine position in ASIA evaluation, the manual muscle testing was performed with the subject either sitting in their own chair or on a standard chair. To minimize the effect of position on test scores, body and limb positions were kept consistent within and between subjects. Individual score for key muscle strength is listed in the Supplementary Appendix (http://links.lww.com/PHM/A470).

\section{Testing of Grip and Pinch Strength}

A hand-held dynamometer (Lafayette Instrument testing range $0-110 \mathrm{lb}$, in $1-1 \mathrm{~b}$ increments) and pinch gauge (B\&L Engineering testing range $0-30 \mathrm{lb}$, in $1-\mathrm{lb}$ increments) were used to measure maximum gross grasp and pinch strength. Scores were averaged for three attempts with each hand. The minimum possible value of $0 \mathrm{lb}$ was assigned when the subject

TABLE 1. Descriptive features of study subjects

\begin{tabular}{|c|c|c|c|c|c|c|}
\hline Subject Number & Dominant Hand, Preinjury & Age, yr & Sex & Duration of Injury, mo & Level of Injury & ASIA Grade \\
\hline 1 & Right & 28 & $\mathrm{~F}$ & 29 & $\mathrm{C} 2$ & $\mathrm{C}$ \\
\hline 2 & Left & 70 & M & 7 & $\mathrm{C} 3$ & $\mathrm{D}$ \\
\hline 3 & Right & 24 & M & 17 & $\mathrm{C} 4$ & $\mathrm{D}$ \\
\hline 4 & Right & 60 & M & 12 & $\mathrm{C} 5$ & $\mathrm{D}$ \\
\hline 5 & Right & 40 & M & 36 & $\mathrm{C} 5$ & $\mathrm{C}$ \\
\hline 6 & Right & 44 & M & 83 & $\mathrm{C} 5$ & $\mathrm{C}$ \\
\hline 7 & Left & 35 & $\mathrm{~F}$ & 191 & C6 & $\mathrm{C}$ \\
\hline 8 & Left & 19 & M & 20 & C6 & $\mathrm{D}$ \\
\hline
\end{tabular}

F, female; M, male. 
could not actively flex the fingers or grasp the dynamometer or pinch meter. ${ }^{17}$

\section{Testing for Arm and Hand Function}

The Jebsen-Taylor Hand Function Test (JTHFT) ${ }^{18}$ is a widely used test with good validity and reliability ${ }^{19}$ and capacity for detecting performance change in activities that resemble daily life activities. It is used to measure the time needed to perform seven everyday activities such as writing, flipping cards, lifting and placing small objects, stacking checkers, feeding, and lifting heavy and light cans. We excluded the writing task, because of heavy dependence on side with less impairment. The subtests are traditionally scored by recording the number of seconds to complete each task. In our study, we choose to discontinue administration of subtests after 180 secs if the subject could not complete the task by that time (maximum $1080 \mathrm{sec}$ with lower scores indicating better performance). In addition, scores for each subtest were recorded in total number of seconds and number of items completed and results were reported as number of items moved per second (items/second). This recording method allowed us to demonstrate change in performance, that is, number of items moved, even though the subject was not able to complete all items within 180 secs.

In addition to JTHFT, the 19-item Action Research Arm Test (ARAT) was used to measure subjects' ability to pick up and release objects of different size and shapes either vertically (30 cm high shelf), horizontally (forward) on the table or to perform whole arm movement by touching the back of head, top of head, and mouth. The test has been standardized by Yozbatiran et al. ${ }^{20}$

As a commonly used measure of function, the JTHFT focuses more on distal part of the upper limb (the hand), whereas the ARAT measures also the contribution of the proximal limb to hand functions by lifting objects onto a shelf $(30 \mathrm{~cm})$ or reaching out from shoulder and elbow to move objects horizontally. Our intervention protocol involved training of both distal and proximal muscle groups, and therefore, we administered two tests. A minimal clinical significant score of 5.7 has been established for ARAT. ${ }^{21}$

\section{Testing of Independence in Daily Activities}

The Spinal Cord Independence Measure II is a disability scale developed specifically for patients with SCI. Its reliability and sensibility to measure changes in function have been well documented. ${ }^{22}$ The total Spinal Cord Independence Measure score ranges from 0 to 100 including the following areas of function: self-care, respiration and sphincter management, and mobility. ${ }^{23}$

\section{Testing for Tolerability}

Tolerability of robotic-assisted training was assessed at each session by questioning the level of perceived pain/ discomfort and fatigue immediately after training and scores were compared with beginning of session. On a visual analog scale, subjects were asked to indicate the level of their perceived fatigue and pain or discomfort. The pain/discomfort scale ranged from 0 indicating no pain/discomfort to 100 indicating worst pain/discomfort possible; fatigue scale ranged from 0 indicating no fatigue to 100 indicating extreme fatigue. ${ }^{24}$ In addition, adverse events were collected.

\section{Description of the Robotic Exoskeleton}

The MAHI Exo-II, a five degree-of-freedom robot, is an electrically actuated upper limb haptic exoskeleton device and has been designed for rehabilitation applications. ${ }^{25,26}$

\section{Description of the Treatment Protocol}

Robotic training was provided with the MAHI Exo-II for 12 sessions for 4 wks. Each session lasted approximately $3 \mathrm{hrs}$ and involved training of both arms. During treatment sessions, subjects sat comfortably in their own chair or in a chair with no arm rest on the side being trained. Knees were flexed and trunk was maintained against the back of the chair, with shoulders slightly abducted and elbows slightly flexed. The hand was secured to the exoskeleton's handle if the subject could not actively grasp and hold the handle. All anatomical joints were aligned with the device's joints. In addition, the height of the device was adjusted to keep the shoulders level to the ground. The subject sat facing the computer monitor (Fig. 1).

Therapy sessions were tailored individually for each joint's movement capability. Single degree-of-freedom movement for elbow, forearm, and wrist was repeated at high intensity. The study subjects were trained in several control modes. A given control mode was selected by the therapist for each movement on each day to maximally engage the subject based on their
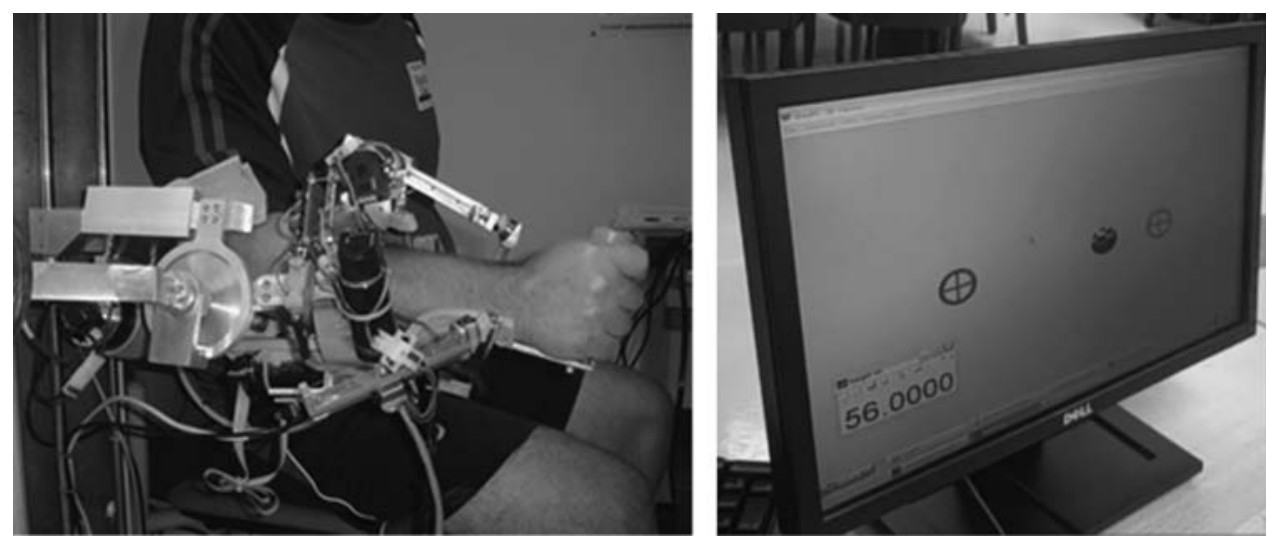

FIGURE 1. MAHI Exo-II robotic device during training (left) and target hitting task (right). 
ability to complete movements. In the subject-passive mode, the MAHI Exo-II moved the patient's limb to the desired target. In the subject-triggered mode, the movement had to be initiated by the subject until a sufficient force was applied in the direction of desired motion to trigger the MAHI Exo-II to carry the limb to the desired target location. In the active-constrained mode, the MAHI Exo-II applied resistive torques to the subject while they moved actively toward the desired target. Actual training time for each side as a proportion of the 3-hr session increased gradually for 4 wks. Treatment was progressed gradually by increasing number of repetitions, by progressing from passive to triggered to active-constrained mode, and by increasing the threshold force in subject-triggered mode or increasing the amount of resistance to be overcome in the active-constrained mode. Graphical feedback about performance was given after each attempt to maintain motivation. Rest breaks were given to avoid fatigue. During the course of therapy, study subjects were not allowed to participate to any other occupational therapy program.

\section{Data Analyses}

Subjects were tested separately for right and left upper limb motor levels between C5 and T1, grip, and pinch strength and for functional level. The scores from each side were summed and analyzed to determine the changes from baseline to immediately after treatment and for 6-mo follow-up. All data are expressed as means(standard error of the mean or SEM). Analyses were performed with nonparametric methods and Wilcoxon signed-rank test to measure differences between baseline and week 4 , in addition to change from baseline to 6-mo poststudy values for all outcome measures $(P<0.05)$.

\section{RESULTS}

Of the 10 subjects enrolled, 8 completed the study. In addition to traumatic cervical SCI, 1 subject had arthritis in thumb, 1 in his left knee, and another subject in his right shoulder. After four sessions, one subject (male, age $=76 \mathrm{yrs}$ ) dropped out from the study because of additional co-morbidity (diabetes mellitus) and the long commute time to and from study site (up to approximately $2 \mathrm{hrs}$ ). A second subject (male, age $=40 \mathrm{yrs}$ ) dropped out after two sessions because of absence of caregiver. Demographic and clinical characteristics of subjects who completed the study are shown in Table 1.

\section{Clinical and Functional Outcomes}

\section{Strength of Selective Muscle Groups}

At baseline, subjects presented varying degrees of impairment of the right and left arm. Based on their baseline manual muscle test scores, only five subjects had higher motor function on their preinjury dominant side, whereas three subjects had lower motor function. As expected, elbow flexion and wrist extension had the highest score on manual muscle testing and little finger abduction the lowest. After treatment, change in muscle strength was highest in elbow extension ( $6 \%$ increase compared with baseline) followed by little finger abduction (15\% increase). After 12 sessions of training, subjects demonstrated significant gains in study endpoints. Gain in total ASIA-UEMS (range $=0-50$ ) was significant after treatment and maintained at 6-mo follow-up (Table 2).

\section{Less Versus More Affected Arm}

Gains in the more affected side were higher (12.2[1.3]14.6[1.5], $P=0.04$, and follow-up (f/u) $=16.1$ [1.4], $P=0.01$ ) compared with the less affected arm (19.1[1.6]-19.4[1.6], $P=0.31$, and $\mathrm{f} / \mathrm{u}=19.5[1.4], P=0.52$ ). Comparison of individual key muscle strength yield significant increase for C7-level elbow extensor immediately after treatment $(2.3[0.6]-3.1[0.5]$, $P=0.04$, and $\mathrm{f} / \mathrm{u}=3.2[0.4], P=0.06)$ and $\mathrm{C} 8$-level finger flexors at 6-mo follow-up (1.8[0.6]-2 [0.59], $P=0.35$, and $\mathrm{f} / \mathrm{u}=3.1[0.6], P=0.04)$ on the more affected side.

\section{Grip and Pinch Force}

Grip force and pinch force demonstrated significant improvement after treatment and were maintained at 6-mo follow-up (Table 2).

\section{Less Versus More Affected Arm}

Gain in grip strength was higher in the more affected arm (3.7 [5.2]-5.6 [7.0], $P=0.04$, and f/u $=4.6[7.6], P=0.26$ ) compared with the less affected arm (15.7[16.5]-18.48[18.07], $P=0.01$, and $\mathrm{f} / \mathrm{u}=21[17.1], P=0.1)$.

Significant gain in pinch strength was observed at follow-up in the more affected arm $(2.7[0.6]-3.1[1.8]$, $P=0.14$, and $\mathrm{f} / \mathrm{u}=3.4[0.6], P=0.02)$ compared with the less affected arm, which showed bigger improvement immediately after treatment (6.4[1.9]-8.2 [2.1], $P=0.01$, and $\mathrm{f} / \mathrm{u}=8.2[1.9]$, $P=0.06)$.

TABLE 2. Arm and hand muscle strength and level of independence in daily activities before and after treatment and at 6-mo follow-up

\begin{tabular}{|c|c|c|c|c|c|c|c|c|}
\hline \multirow[b]{2}{*}{ Measurement (Range of Scores) } & \multicolumn{2}{|c|}{ Baseline } & \multicolumn{3}{|c|}{ Post-treatment } & \multicolumn{3}{|c|}{ 6-mo Follow-up (Pre-treatment) } \\
\hline & Mean (SEM) & Min-Max & Mean (SEM) & Min-Max & $\boldsymbol{P}$ & Mean (SEM) & Min-Max & $\boldsymbol{P}$ \\
\hline ASIA-UEMS (0-50) & $31.5(2.3)$ & $24-44$ & $34(2.3)$ & $24-44$ & 0.04 & $35.5(2)$ & $28-43$ & 0.02 \\
\hline Grip force, $\mathrm{lb}$ & $9.7(3.8)$ & $0-30.2$ & $12(4.3)$ & $0-34.8$ & 0.02 & $12.7(4)$ & $0-33.9$ & 0.05 \\
\hline Pinch force, $\mathrm{lb}$ & $4.5(1.1)$ & $0-10$ & $5.7(1.2)$ & $0-11.9$ & 0.01 & $5.6(1.2)$ & $0-11.9$ & 0.02 \\
\hline SCIM II & $62.1(9.7)$ & $26-93$ & $62.6(9.7)$ & $26-93$ & 0.18 & $67.6(9.5)$ & $33-95$ & 0.07 \\
\hline
\end{tabular}

Scores from right and left upper limb are averaged. $P$ value reflects comparison via Wilcoxon signed-rank test between baseline and end of treatment and between baseline and 6-mo follow-up. 
TABLE 3. The JTHFT at baseline and after treatment and at 6-mo follow-up

\begin{tabular}{|c|c|c|c|c|c|c|c|c|}
\hline JTHFT Subtests & \multicolumn{2}{|c|}{ Baseline } & \multicolumn{3}{|c|}{ Post-treatment } & \multicolumn{3}{|c|}{ 6-mo Follow-up (Pre-treatment) } \\
\hline Page turning & $0.23(0.05)$ & $0.02-0.43$ & $0.31(0.08)$ & $0.03-0.68$ & 0.02 & $0.36(0.09)$ & $0.03-0.77$ & 0.02 \\
\hline Simulated feeding & $0.17(0.06)$ & $0.02-0.39$ & $0.27(0.08)$ & $0.03-0.53$ & 0.07 & $0.28(0.08)$ & $0.03-0.56$ & 0.04 \\
\hline Stacking checkers & $0.11(0.3)$ & $0.02-0.27$ & $0.19(0.05)$ & $0.02-0.45$ & 0.03 & $0.25(0.08)$ & $0.02-0.57$ & 0.06 \\
\hline Lifting light cans & $0.20(0.07)$ & $0.03-0.53$ & $0.32(0.11)$ & $0.03-0.88$ & 0.05 & $0.39(0.14)$ & $0.03-1.06$ & 0.06 \\
\hline Total & $0.14(0.04)$ & $0.02-0.41$ & $0.21(0.07)$ & $0.03-0.60$ & 0.04 & $0.29(0.25)$ & $0.03-0.63$ & 0.07 \\
\hline
\end{tabular}

Values are mean (SEM) change from baseline to end of therapy and to 6-mo follow-up. Scores from right and left upper limb are averaged, and $P$ values reflect comparison via Wilcoxon signed-rank test between baseline and end of treatment and between baseline and 6-mo follow-up. Scores indicate number of items moved within $1 \mathrm{sec}$.

\section{Arm and Hand Function}

Two outcome measures for arm and hand functions, the JTHFT and the ARAT, showed significant improvement in total score (Table 3, Fig. 2). Some improvements in function were maintained at 6 mos after training. In three subjects, gains in ARAT score in one arm function exceeded the minimal clinical significant difference of 5.7 points after treatment, and at 6-mo follow-up, the number of subjects who exceeded the minimal clinically significant difference score (compared with baseline) increased to four. Of those, two subjects showed significant change on both arms, and two subjects had significant change on one arm.

\section{Less Versus More Affected Arm}

Both arms showed significant improvement after treatment and at follow-up. However, the change in the less affected arm was higher $(0.34[0.06]-0.45[0.09], P=0.01$, and $\mathrm{f} / \mathrm{u}=0.0 .45[0.08], P=0.02)$ compared with the more affected arm $(0.10[0.04]-0.17[0.06], P=0.05$, and $\mathrm{f} / \mathrm{u}=0.21$ [0.08], $P=0.06)$.

\section{Less Versus More Affected Arm}

Both arms showed improvement after treatment, but the change in the less affected arm was higher from before to after treatment (42.7 [4.7]-46.9[4.6], $P=0.04$, and f $/ \mathrm{u}=46.9$ [3.8], $P=0.03)$ compared with the more affected arm $(18.7[5.1]$ $21.7[5.4], P=0.1$, and $\mathrm{f} / \mathrm{u}=25$ [4.9], $P=0.04)$. In addition, after intervention, change in ARAT score in two subjects reached the minimal clinically significant difference of 5.7 points for the less affected arm, and one subject reached this difference in the more affected arm. At 6-mo follow-up, two subjects reached minimal clinically significant difference in both arms, one subject in the less affected arm and one subject in the more affected arm.

\section{Quality of Life}

Changes in Spinal Cord Independence Measure II scores are shown in Table 2. No statistically significant difference was found after treatment or at follow-up when compared with baseline.

\section{Tolerability}

Subjects' self-report on pain and discomfort level did not show any significant increase during therapy sessions. Level of fatigue showed slight increases after each session, but no therapy session was missed or had to be rescheduled because of the previously mentioned symptoms.

\section{DISCUSSION}

The primary goals of this proof-of-concept study were to evaluate functional benefits of a treatment program developed to improve arm and hand motor functions and to assess tolerability and feasibility of MAHI Exo-II as an adjunct modality for upper limb rehabilitation in chronic, cervical SCI.

The main study result has demonstrated that adults with chronic tetraplegia can benefit from intensive, repetitive training

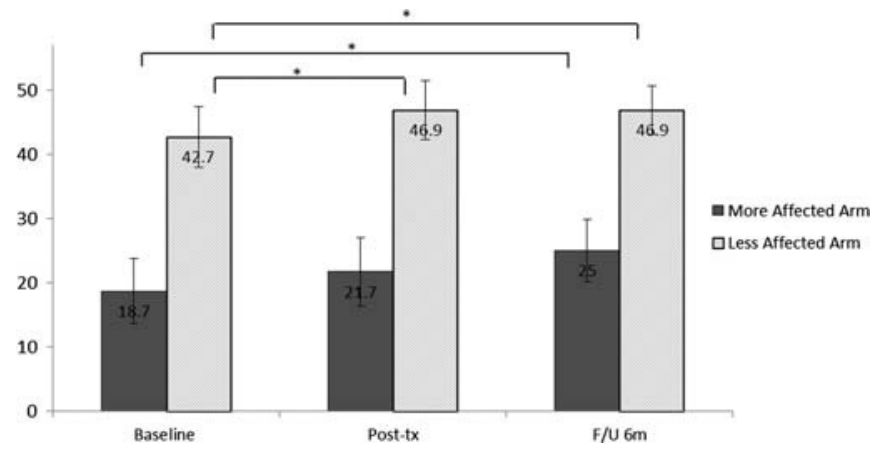

FIGURE 2. The ARAT score at baseline, immediately after treatment, and at 6-mo follow-up after robotic-assisted training. 
and that some improvement in arm and hand function was persistent at 6 mos after the end of intervention. However, results should be interpreted with caution because the pilot study lacked a control group and had a small number of patients. Therefore, no conclusion should be drawn without a welldesigned randomized controlled clinical trial. Nevertheless, on an individual basis, the results are encouraging for several reasons. First, all participants were at chronic stage of their injury. Second, research on arm and hand functioning in chronic, cervical SCI is relatively limited. Individuals with incomplete tetraplegia have residual motor function below injury level, and rehabilitation strategies should focus less on compensation and more on activity-based approaches to improve skilled movement performance. In the previous decades, both animal and human studies have provided sufficient evidence that sensorimotor function after SCI is enhanced by facilitation of neuroplasticity through functional training, 26,27 and repetitive, intense training is the main factor in promoting such plastic changes in the central nervous system. ${ }^{9,28}$ The present results support the hypothesis that active repetitive movement of arm and wrist when coupled with exertion from the subject to overcome resistive forces produced by the robot may generate clinical benefits.

The dose for robotic training was set by a highly trained and experienced physical therapist experimentally, by varying the control mode of the robot, the amount of resistance generated by the robot, and the number of repetitions at each session. Consequently, subjects were challenged to perform at a higher intensity compared with previous sessions. On average, the number of repetitions gradually progressed from approximately 200 repetitions on day 1 to approximately $1000-1500$ on day 12 . The progress was tailored to each subject's physical tolerance level for a given movement. Despite long training duration ( 3 hrs of contact time with up to 60-70 mins of active training time per limb), roboticassisted training was feasible and highly tolerable (90\%). Compliance with intervention protocol was at $100 \%$. Subjects did not miss any therapy session due to excessive fatigue or soreness in upper limb or trunk/neck muscles. The task was easy and the feedback was useful to maintain motivation. In addition, there was no report of other adverse events related to overuse of musculoskeletal system such as severe pain or discomfort in the joints and surrounding soft tissue due to excessive forces acting upon shoulder girdle or elbow and wrist. In this case, MAHI Exo-II is designed to fit well with anatomical joints and its passive degree-of-freedom along the longitudinal axes at wrist and hand enables easy positioning of various arm lengths. In addition, optimal positioning of subjects before training and constant monitoring during training was ensured.

These resultant behavioral gains highlight the importance of repetitive, active training. The specific factors that contributed most to the measured gains remain unclear; however, potential mechanisms may include activity-dependent neuroplastic changes, peripheral muscle strengthening, particularly increased strength of wrist extensors with resultant tenodesis effect, and improved muscle endurance.

An interesting finding was that after 4 wks of intervention, gain from the repetitive training could be extended in overall arm function, as demonstrated with function-based assessments such as JTHFT and ARAT. Change in ARAT score in three subjects had even reached minimal clinically significant difference of 5.7 points after treatment, and this was maintained at 6-mo follow-up. Generalization has been demonstrated in similar studies with stroke patients using robotic-assisted training as intervention. ${ }^{10}$ The findings from these studies have shown that patients undergoing repetitive hand flexion/extension movement training showed improvements not only in the trained distal part of the limb (i.e., forearm and wrist movements) but overall in the hand and arm activities. However, these activities were not translated into more complex function of arm and hand as measured by the Spinal Cord Independence Measure II. In self-report, subjects required less assistance in activities such as lower body dressing, picking and releasing small objects, grasping, hugging, and turning pages. Results from this pilot study were consistent with our findings previously reported for a singlecase study. ${ }^{14}$

\section{CONCLUSIONS}

Despite the study's limitations, the findings demonstrate that robotic-assisted training of arm and hand functions after high level SCI can be an adjunctive rehabilitation method. A necessity exists for performing larger controlled studies to further evaluate the tolerability, feasibility, and efficacy of using robotic training in the future.

\section{REFERENCES}

1. Anderson KD: Targeting recovery: priorities of the spinal cord-injured population. J Neurotrauma 2004;21:1371-83

2. Snoek GJ, IJzerman MJ, Hermens HJ, et al: Survey of the needs of patients with spinal cord injury: impact and priority for improvement in hand function in tetraplegics. Spinal Cord 2004;42:526-32

3. Popovic MR, Kapadia N, Zivanovic V, et al: Functional electrical stimulation therapy of voluntary grasping versus only conventional rehabilitation for patients with subacute incomplete tetraplegia: a randomized clinical trial. Neurorehabil Neural Repair 2011;25:433-42

4. Ying Z, Roy RR, Edgerton VR, et al: Exercise restores levels of neurotrophins and synaptic plasticity following spinal cord injury. Exp Neurol 2005;193:411-9

5. Kilgore KL, Hoyen HA, Bryden AM, et al: An implanted upper-extremity neuroprosthesis using myoelectric control. J Hand Surg 2008;33:539-50

6. Ortner R, Allison BZ, Korisek G, et al: An SSVEP BCI to control a hand orthosis for persons with tetraplegia. IEEE Trans Neural Syst Rehabil Eng 2011;19:1-5

7. Hamou C, Shah NR, DiPonio L, et al: Pinch and elbow extension restoration in people with tetraplegia: a systematic review of the literature. $J$ Hand Surg 2009;34:692-9

8. Dietz V, Müller R, Colombo G: Locomotor activity in spinal man: significance of afferent input from joint and load receptors. Brain 2002;125(Pt 12):2626-34

9. Beekhuizen KS, Field-Fote EC: Massed practice versus massed practice with stimulation: effects on upper extremity function and cortical plasticity in individuals with incomplete cervical spinal cord injury. Neurorehabil Neural Repair 2005; $19: 33-45$

10. Takahashi CD, Der-Yeghiaian L, Le V, et al: Robot-based hand motor therapy after stroke. Brain 2008;131(Pt 2):425-37

11. Hornby TG, Zemon DH, Campbell D: Robotic-assisted, body-weight-supported treadmill training in individuals following motor incomplete spinal cord injury. Phys Ther 2005;85:52-66

12. Field-Fote EC, Lindley SD, Sherman AL: Locomotor training approaches for individuals with spinal cord injury: a preliminary report of walking-related outcomes. J Neurol Phys Ther 2005;29:127-37

13. Zariffa J, Kapadia N, Kramer JL, et al: Effect of a robotic rehabilitation device on upper limb function in a sub-acute cervical spinal cord injury population. IEEE Int Conf Rehabil Robot 2011;2011:5975400

14. Yozbatiran N, Berliner J, O'Malley MK, et al: Robotic training and clinical assessment of upper extremity movements after spinal cord injury: a single case report. $J$ Rehabil Med 2012;44:186-8

15. Cortes M, Elder J, Rykman A, et al: Improved motor performance in chronic spinal cord injury following upper-limb robotic training. NeuroRehabilitation 2013;33:57-65 
16. Steeves JD, Lammertse D, Curt A, et al: Guidelines for the conduct of clinical trials for spinal cord injury (SCI) as developed by the ICCP panel: clinical trial outcome measures. Spinal Cord 2007;45:206-21

17. Mathiowetz V, Weber K, Volland G, et al: Reliability and validity of grip and pinch strength evaluations. J Hand Surg Am 1984;9:222-6

18. Jebsen RH, Taylor N, Trieschmann RB, et al: An objective and standardized test of hand function. Arch Phys Med Rehabil 1969;50:311-9

19. Sears ED, Chung KC: Validity and responsiveness of the Jebsen-Taylor Hand Function Test. J Hand Surg Am 2010;35:30-7

20. Yozbatiran N, Der-Yeghiaian L, Cramer SC: A standardized approach to performing the action research arm test. Neurorehabil Neural Repair 2008;22:78-90

21. van der Lee JH, Beckerman H, Lankhorst GJ, et al: The responsiveness of the Action Research Arm test and the Fugl-Meyer Assessment scale in chronic stroke patients. $J$ Rehabil Med 2001;33:110-3

22. Wirth B, van Hedel HJ, Kometer B, et al: Changes in activity after a complete spinal cord injury as measured by the Spinal Cord Independence Measure II (SCIM II). Neurorehabil Neural Repair 2008;22:279-87
23. Catz A, Itzkovich M, Agranov E, et al: The spinal cord independence measure (SCIM): sensitivity to functional changes in subgroups of spinal cord lesion patients. Spinal Cord 2001;39:97-100

24. New PW, Lim TC, Hill ST, et al: A survey of pain during rehabilitation after acute spinal cord injury. Spinal Cord 1997;35:658-63

25. Fitle KD, Pehlivan AU, O'Malley MK: A robotic exoskeleton for rehabilitation and assessment of the upper limb following incomplete spinal cord injury. Paper presented at: 2015 IEEE International Conference on Robotics and Automation (ICRA); May 26-30, 2015; Seattle, WA

26. Behrman AL, Bowden MG, Nair PM: Neuroplasticity after spinal cord injury and training: an emerging paradigm shift in rehabilitation and walking recovery. Phys Ther 2006; $86: 1406-25$

27. Dietz V, Fouad K: Restoration of sensorimotor functions after spinal cord injury. Brain 2014;137(Pt 3):654-67

28. Hoffman LR, Field-Fote EC: Cortical reorganization following bimanual training and somatosensory stimulation in cervical spinal cord injury: a case report. Phys Ther 2007;87:208-23 